\title{
EDITORIAL
}

\section{Tumour response, correlates of survival and clinical benefit}

W e are all familiar with the metrics of tumour shrinkage and time to the development of disease progression as important end points in clinical trials. This tenet is based on findings of numerous studies over several decades that have demonstrated a link between agents that cause tumour shrinkage in a cancer population and its correlation with an overall survival improvement. The problem is that the inevitable variation in how these definitions and criteria have been applied over many decades in clinical trials has led to different conclusions about the efficacy of a treatment. Added to this complexity are the following issues: nonmeasurable lesions, differences obtained with the method of assessment of progression (imaging versus clinical examination), changes in non-target lesions versus target lesions, impact of lesions that coalesce or split on treatment, to name but a few. When considering the additional complexities posed by these factors, it is perhaps not surprising that tumour response is often a poor indicator of survival outcome. Yet, how can this be reconciled to allow the field of oncology to move forward.

Disease progression is considered to represent a detrimental change in tumour burden. However, it is possible that increased tumour burden might not represent clinically detrimental disease progression. Conversely, a lack of change in tumour burden might not signal an absence of disease progression. Our existing definition of tumour response is a measure that describes what happens to a tumour during therapy, as noted by Eisenhauer and co-authors in the revised RECIST criteria version 1.1 .

So, in what circumstances can tumour response be considered a reliable biomarker of a survival outcome? One suggestion is if the reduction in tumour shrinkage of measurable disease in relation to the overall size of the tumour (before treatment) is enough to provide clinical benefit to the patient, in terms of symptom control, perhaps this can be considered a reasonable correlate? The next question is how we define the cut-off for this measure? This is a difficult metric to set a threshold for, because the tumour can constantly change in size and location during its disease trajectory. Nevertheless, if a lack of disease progression seems to be associated with symptom control, it is interesting to speculate on the idea of symptom control as a surrogate for disease progression or stable disease in clinical trials. Symptom control, however, is a subjective measure, and so would be considered less comparable and robust as a trial end point. Despite these issues, if physicians could show that quantitative measurement of a symptom could be just as good a surrogate as RECIST-measured response criteria in a clinical trial, then regulatory bodies might well consider this as an accompanying surrogate end point or even a sufficient measure alone by which to consider a drug or treatment approval.

We know that tumour shrinkage with some targeted agents has been so impressive, one would almost consider the patient cured, and yet months later the resistant subclones return and the patient relapses. In this example and at the biological level, how does tumour response and its regrowth relate to survival? Unfortunately, the relationship is poor and tumour response is almost an indirect surrogate for ensuing disease progression. Thus, in the era of heightened awareness of disease resistance, tumour and normal tissue heterogeneity, the term 'clinical benefit' is even more pertinent. So, how do we define clinical benefit? Intuitively, one might assume this metric is a patient-defined measure. Essentially, clinical benefit includes those individuals who have achieved an objective response and those who do not seem to have cancers that have progressed for a prospective-trial-specified period. Yet, this end point is still defined by physicians using objective-based metrics that have a degree of convenience, and that are reliable and reproducible-rather than something patients regard as 'clinically beneficial'. Moreover, the parameters of clinical benefit should be placed also in the context of the patient's prior health and their function at baseline, before they received the treatment-a consideration often overlooked in trials. Furthermore, it is important to delineate the natural history of the disease as the tumour progresses from the effect a regimen might have on the patient's cancer, an effect rarely ascertained in trials owing to a lack of appropriate controls or assessment of the surrounding noncancerous tissue. Crucially, we need to define response in a patient-centric way and correlate that response with the biology of the tumour rather than using measurable end points because they are convenient.

In terms of a way forward, although we should not throw out the baby with the bathwater and abandon our historical trial end points that have provided notable advances, we do need to employ greater scrutiny in gathering evidence for how we define response in relation to disease progression and patient relapse. We owe it to our patients to set the bar higher for end point definition so we can better correlate response with survival.

doi:10.1038/nrclinonc.2015.134

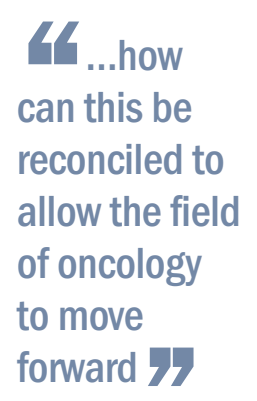

Lisa Hutchinson is the Chief Editor of Nature Reviews Clinical Oncology.

Competing interests The author declares no competing interests. 